\title{
Controlled angular redirection of light via nanoimprinted disordered gratings
}

Buss, Thomas; Teisseire, Jérémie; Mazoyer, Simon; Smith, Cameron; Mikkelsen, Morten Bo Lindholm; Kristensen, Anders; Søndergård, Elin

\section{Published in:}

Applied Optics

Link to article, DOI:

10.1364/AO.52.000709

Publication date:

2013

Document Version

Publisher's PDF, also known as Version of record

Link back to DTU Orbit

Citation (APA):

Buss, T., Teisseire, J., Mazoyer, S., Smith, C., Mikkelsen, M. B. L., Kristensen, A., \& Søndergård, E. (2013). Controlled angular redirection of light via nanoimprinted disordered gratings. Applied Optics, 52(4), 709-716. https://doi.org/10.1364/AO.52.000709

\section{General rights}

Copyright and moral rights for the publications made accessible in the public portal are retained by the authors and/or other copyright owners and it is a condition of accessing publications that users recognise and abide by the legal requirements associated with these rights.

- Users may download and print one copy of any publication from the public portal for the purpose of private study or research.

- You may not further distribute the material or use it for any profit-making activity or commercial gain

- You may freely distribute the URL identifying the publication in the public portal 


\title{
Controlled angular redirection of light via nanoimprinted disordered gratings
}

\author{
Thomas Buß, ${ }^{1}$ Jérémie Teisseire, ${ }^{2}$ Simon Mazoyer, ${ }^{3}$ Cameron L. C. Smith, ${ }^{1}$ \\ Morten Bo Mikkelsen, ${ }^{1}$ Anders Kristensen, ${ }^{1}$ and Elin Søndergård ${ }^{2, *}$ \\ 'DTU Nanotech, Department of Micro and Nanotechnology, Technical University of Denmark, Building 345 East, \\ Kongens Lyngby DK-2800, Denmark \\ ${ }^{2}$ Surface du Verre et Interfaces, UMR 125 CNRS/Saint-Gobain, 39, quai Lucien Lefranc, \\ Aubervilliers Cedex F-93303, France \\ ${ }^{3}$ Saint-Gobain Recherche, 39, quai Lucien Lefranc, Aubervilliers Cedex F-93303, France \\ ${ }^{*}$ Corresponding author: Elin.Sondergard@saint-gobain.com \\ Received 18 September 2012; accepted 1 November 2012; \\ posted 14 November 2012 (Doc. ID 176486); published 30 January 2013
}

\begin{abstract}
Enhanced control of diffraction through transparent substrates is achieved via disordered gratings in a silica sol-gel film. Tailoring the degree of disorder allows tuning of the diffractive behavior from discrete orders into broad distributions over large angular range. Gratings of optical quality are formed by silica sol-gel nanoimprint lithography and an optical setup for the measurement of continuous diffraction patterns is presented. Sound agreement is found between measurements and simulation, validating both the approach for redirection of light and the fabrication process. The disordered gratings are presented in the context of improved interior daylighting and may furthermore be suited to a wide variety of applications where controlled angular redirection of light is desired. (C) 2013 Optical Society of America OCIS codes: $\quad$ 050.1950, 080.4298, 220.4241, 310.6628, 120.0120.
\end{abstract}

\section{Introduction}

Optically functional surfaces can be fabricated by patterning the surface of a substrate with microand nanostructures [1-3]. Among such surface structures, diffraction gratings [4] are key optical elements for controlled redirection of light, which allow the design of highly compact optical systems $[5,6]$. Due to their strongly dispersive nature, diffraction gratings are most often used when monochromatic light is involved or when spectral separation is required [7], but this renders them typically ill-suited to purposes where any divergence of color is unwanted. However, diffraction gratings can address this issue by introducing a degree of disorder to the grating properties [8-10], such that the diffraction angle of each wavelength is broadened. In this work, varying degrees

$1559-128 \mathrm{X} / 13 / 040709-08 \$ 15.00 / 0$

(C) 2013 Optical Society of America of disorder to grating periods are implemented, yielding an additional degree of freedom in the design of a desired diffraction pattern such that the redirection of the light through a transmission grating can be controlled.

The findings are presented in the context of daylighting $[11,12]$ through windows, i.e., optimizing the path of sunlight for improved natural indoor lighting, although the method can be generalized and adapted to a number of applications that include color-controlled lighting in optical systems [13] and optical sensing [14].

For use of diffraction gratings as optically functional surface structures in windows with optimized daylighting functionality, an additional constraint is imposed by the fact that the grating structures need to be fabricated over a large area in a material with specific optical and mechanical properties. This can be achieved by using a silica sol-gel nanoimprint lithography (SSGNIL) process, where nanostructures 
are defined in a thin film of silica gel with a stamp [15]. Such a process enables cheap fabrication of silica nanostructures on large surfaces, and has potential for large-scale [16] production.

The layout of the paper is structured in the following way: in Section 2, the principle of operation and the requirements for the specific use of disordered gratings as an optical daylighting device are discussed. In Section 3 , the method used to simulate the diffraction characteristics of the disordered gratings, in order to select grating designs for fabrication and measurements, is presented. Section 4 outlines the fabrication process for making silica patterns on glass substrates, and Section $\underline{5}$ describes in detail the optical setup, developed to measure continuous diffraction patterns over large angles. Optical measurements demonstrating that broad control of light redirection can be obtained by tailoring the amount of disorder in the gratings are reported in Section $\underline{6}$, and the measured and simulated efficiencies are related to the quality of the fabrication process. Compelling qualitative agreement between simulation and measured data is observed. Finally, a conclusion is drawn from the results and their implications, both for daylighting and other potential applications where customizable diffraction patterns are of interest, are discussed. It is thereby shown that surface patterning of engineered disordered gratings via SSGNIL can yield new optical functionality.

\section{Principle of Operation}

Windows with daylighting functionality improve the global luminosity of natural light inside a room by spreading sunlight, as it passes through the window, such that part of the light is redirected to the ceiling and further into the room $[17,18]$. This is shown in Fig. 1(a), which illustrates the light distribution in a room with a normal window: a small section of the room is intensely illuminated with direct sunlight while the majority of the room is shadowed. Figure 1(b) shows how this can be improved with a daylighting window. In this case, a more homogeneous light distribution throughout the room is possible by redirecting a portion of the light both horizontally into the room and also toward the ceiling, where it undergoes diffuse scattering. Daylighting functionality can be obtained in many different ways [19], but for practical and architectural reasons, it can be convenient to integrate the functionality directly on the glass of the window, e.g., by applying diffractive optical structures to the surface.

For a daylighting window element to function sufficiently, not only should the absolute transmittance through the window be optimized, but also the spatial and spectral distributions of light are important for comfort and general use [20,21]. However, since typical uniform-period diffraction gratings redirect light into distinct orders, "rainbow effects" appear and render such gratings unsuitable for daylighting purposes. Chirped gratings, where the grating period undergoes a linear variation, are well known for

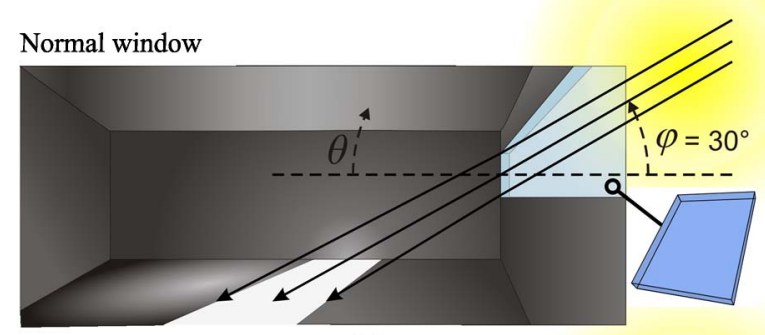

(a)

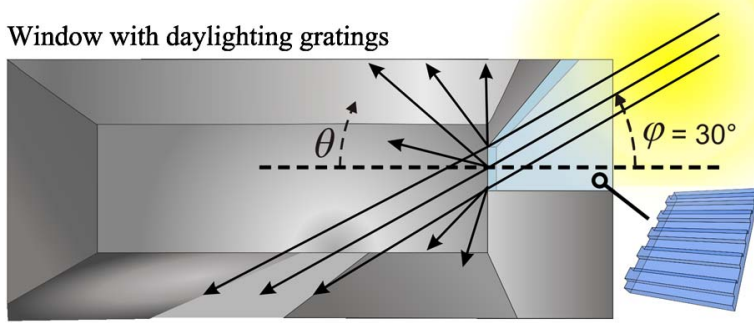

(b)

Fig. 1. (Color online) (a) Illustration of light distribution inside a room with regular, unstructured windows. (b) Daylighting windows comprising, e.g., disordered gratings cause the light to spread more homogenously throughout the room and toward the ceiling. This results in an improved interior lighting during daylight hours. The angles of incident and transmitted light are denoted $\varphi$ and $\theta$, respectively, both measured from the horizontal. Figure adapted from [32].

broadening the diffractive spectrum [22], but the feasible scale of their implementation is orders of magnitude smaller than the size of a typical window pane. Nevertheless, all these issues can be overcome via diffraction gratings with deterministic degrees of disorder, where light can be controllably redirected over a large angular distribution and, by using the right mixture of disorder, done so in a color divergence-free manner.

\section{Design and Simulations}

The design of the daylighting structure is based on a binary grating of period $\Lambda=400 \mathrm{~nm}$ with a filling factor of $50 \%$. Such a structure redirects visible light into a single (-1st) diffraction order. This combines two important properties for a daylighting device in the facade of a building: (i) the majority of light remains present in the 0th order such that the window remains transparent and (ii) a significant fraction of the incident light is diffracted to the ceiling, allowing distributed illumination of the room with natural light. However, in this case for gratings of uniform period, each wavelength is redirected at a specific angle, causing the "rainbow effect." For aesthetical reasons, this should be suppressed such that the light diffracted to the ceiling appears white. In order to achieve this, disorder is introduced into the otherwise uniform gratings to redirect monochromatic light over a larger angular range. Subsequently, the degree of disorder becomes an important parameter of such a system. On the one hand, if the disorder is 
too prominent, the daylighting effect vanishes as the system becomes equivalent to an omnidirectional diffuser and transparency is lost. On the other hand, if the disorder is too low, the "rainbow effect" is not sufficiently suppressed. In order to describe the disorder, the parameter $M$ is used as shown in Fig. 2(a). Starting from a uniform grating of period $\Lambda$, blocks of $M$ periods are kept intact. The disorder is introduced by randomly inserting separations of $\{-\Lambda / 2 ; 0 ;+\Lambda / 2\}$ to the adjacent block. Importantly, the transformation conserves energy, i.e., the total light quantity transmitted by the structure is equivalent to the sum of the quantity diffracted in the 0th and -1 st orders of the uniform grating. Based on the $M$ parameter, the disorder percentage is defined as

$$
\xi=1 /(2 M) .
$$

Since the grating line width is constant, the disorder is limited to $50 \%$ for the case of $M=1$ where a shift is possible after each period. As a figure of merit for the

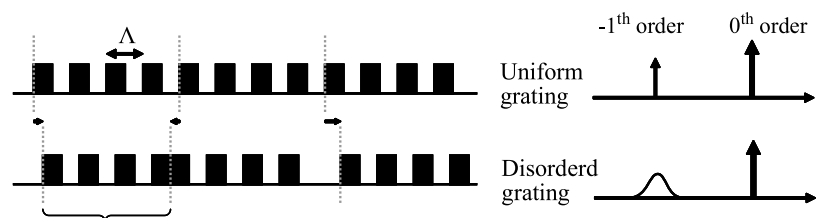

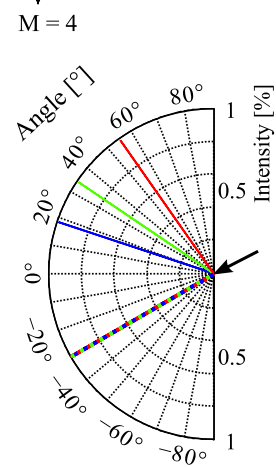

(b) (a)

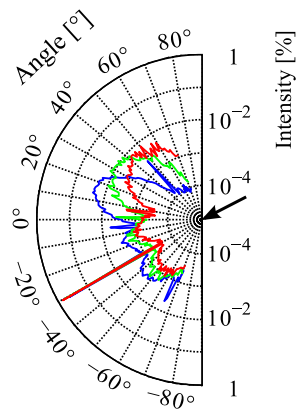

(c)

Design A ( $M=4, \Lambda=400 \mathrm{~nm}, \xi=12.5 \%) \quad$ Design C $(\mathrm{M}=2, \Lambda=500 \mathrm{~nm}, \xi=25 \%)$

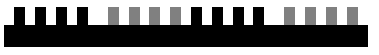

Design $\mathrm{B}(\mathrm{M}=2, \Lambda=400 \mathrm{~nm}, \xi=25 \%)$

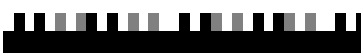

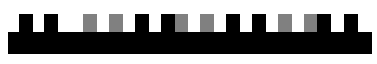

Design D (M = 1, $\Lambda=400 \mathrm{~nm}, \xi=50 \%)$

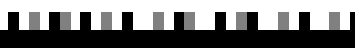

(d)
Fig. 2. (Color online) (a) Disordered gratings are used in order to achieve broadening of the nonzero order diffraction peaks. For every $M$ periods, a random shift of $\{-\lambda / 2 ; 0 ;+\lambda / 2\}$ is inserted. (b) Diffraction of a $500 \mathrm{~nm}$ periodic grating for light incident at $30^{\circ}$ from the surface normal. The 0 th and -1 st diffraction order for light of wavelengths 410,532 , and $661 \mathrm{~nm}$ are shown with peak positions at approximately $19^{\circ}, 33^{\circ}$, and $55^{\circ}$, respectively. (c) The same grating with disordered period $(\xi=25 \%)$ leads to broadening of the peaks around the locations for the uniform grating. (d) Cross-sectional drawings (to scale) of the different grating designs which are investigated. For illustration, adjacent blocks of $M$ periods are shown in black/gray color. The height of the grating lines is $350 \mathrm{~nm}$. effect of the grating, the daylighting efficiency is defined as

$$
\eta_{\text {daylighting }}=\int_{0^{\circ}}^{90^{\circ}} I(\theta) \mathrm{d} \theta / \int_{-90^{\circ}}^{90^{\circ}} I(\theta) \mathrm{d} \theta,
$$

where $\theta$ is the angle and $I(\theta)$ the light irradiance. The daylighting efficiency $\eta_{\text {daylighting }}$ is thus calculated as the ratio of the power diffracted above horizontal over the total transmitted power, and this value is the essential parameter used to characterize the structures in simulations and optical measurements.

In addition to disorder, the optical properties are also affected by the height of the patterns $(h)$ and the period of the grating $(\Lambda)$. In order to test the influence of these parameters, numerical simulations are performed. A rigorous coupled wave analysis (RCWA) calculation method $[23,24]$ is used which allows simulation of periodic structures. In order to adapt it for simulation of the disorder, super-cells of 100 periods are defined that are periodically replicated. Each of these super-cells contains a specific realization of disorder; accordingly, the calculation results are averaged over 20 different realizations of the same $M$ value.

For an actual window with improved daylighting properties made by diffraction gratings, the diffraction will depend on both the elevation and azimuth angle of the sun. In this paper, all simulations and measurements are performed with the sun's azimuth perpendicular to the glass pane and an elevation angle of $\varphi=30^{\circ}$. This angle is chosen since it is large enough for the sun to have high intensity, yet small enough for the sun to cause glare far into a room.

The effect of the disorder is demonstrated in Figs. 2(b) and 2(c). Figure 2(b) shows the diffraction angles of a regular periodic grating and Fig. 2(c) shows simulated data of a disordered grating of the same period, where a broad distribution of light around the peak positions of the uniform grating is observed.

The period of the gratings are chosen to be 400 and $500 \mathrm{~nm}$ in order to operate at visible light wavelengths in the range of 400-700 nm. The effect of the structure height is critical for the daylighting performance, and simulated data are presented in Table 1. Based on these results, the pattern height is kept constant and equal to $350 \mathrm{~nm}$ and should ensure an efficiency of approximately $25 \%$.

Based on the numerical simulations, four different grating designs with different period and disorder parameters have been selected for fabrication. The

Table 1. Calculated Daylighting Efficiency of a $400 \mathrm{~nm}$ Grating with $M=2$ for Different Heights of the Grating Lines

\begin{tabular}{lc}
\hline Grating Height $(\mathrm{nm})$ & Daylighting Efficiency $(\%)$ \\
\hline 100 & 2.4 \\
250 & 14.3 \\
350 & 26.0 \\
\hline
\end{tabular}


effect of disorder is investigated from $12.5 \%$ to $50 \%$ and the influence of the period from 400 and $500 \mathrm{~nm}$ is compared. A drawing of the four structures is shown in Fig. 2(d).

\section{Nanoimprint Fabrication}

In order to fabricate gratings with deterministic disorder, a direct-writing fabrication method is required since self-assembly or holographic approaches are not applicable. The technique in this work is based on replication of a master, which can be easily upscaled to large areas and is thereby cost-effective. The dimensions necessary for daylighting applications also pose a challenge, requiring a period of $400 \mathrm{~nm}$, an aspect ratio of $2 \cdot h / \Lambda=1.75$, and transparency of the material. To fulfill these demands, the simple and low-cost SSGNIL [25,26] process is used to imprint nanometric patterns in a thin film on glass. In particular, the chosen sol-gel material has two principal advantages: its optical properties are similar to glass (refractive index $=1.45$ ) and it has a strong adhesion to glass substrates.

The process consists of mixing of a sol, spincoating, imprinting, and finally demolding, as illustrated in Fig. 3 . For the experiment, $3.6 \mathrm{~g}$ of methyltriethoxysilane (MTES $-0.1 \mathrm{~mol}$ ) are mixed with $5.1 \mathrm{~g}$ of $\mathrm{HCl}(\mathrm{pH}=2.5)$ and stirred for $3 \mathrm{~h}$. The sol is spin-coated (2500 rpm, $30 \mathrm{~s})$ to produce an $800 \mathrm{~nm}$ thin film on a smooth glass substrate. Next, a polydimethylsiloxane stamp (PDMS, Sylgard 184, Dow Corning) is applied onto the spin-coated film with a force of $50 \mathrm{~N}$ (stamp area: $4 \times 3 \mathrm{~cm}^{2}$ ) at $80^{\circ} \mathrm{C}$ for $60 \mathrm{~min}$. During this time, the material fills the cavity of the stamp and cross-links until it reaches a solid state. The temperature is then decreased to ambient temperature, the load is relaxed, and the stamp is removed from the surface, leaving a pattern with the negative shape of the stamp. Details of the SSGNIL process are described in [15].

In order to facilitate imprinting of the high aspect ratio grating lines, the stamp is made as a two-layer structure: The nanostructures are made by casting $h$-PDMS ("hard" PDMS) from a silicon master with grating height of $350 \mathrm{~nm}$, fabricated at CEA-LETI (Grenoble, France) by $e$-beam lithography, and the bulk of the stamp is made in soft PDMS [27]. A thin layer of $h$-PDMS (ABCR, Germany) is first poured onto the master, placed under vacuum to remove possible entrapped bubbles $(1 \mathrm{~h})$, and cured at $60^{\circ} \mathrm{C}$ for $15 \mathrm{~min}$. Following this, a thick layer of PDMS (Sylgard 184) is added directly on the $h$-PDMS layer

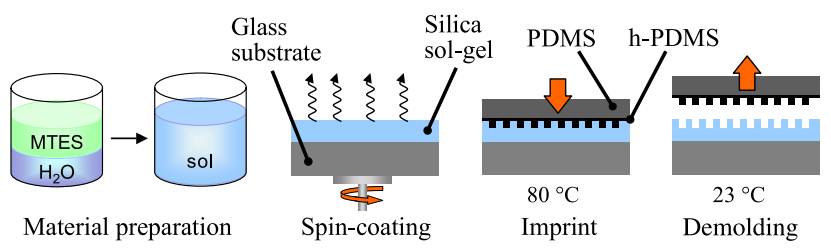

Fig. 3. (Color online) Fabrication process, consisting of sol-gel preparation, spin-coating of the thin-film, imprinting, and demolding. and cured for $2 \mathrm{~h}$ at $60^{\circ} \mathrm{C}$. The resulting surface pattern of the stamp is formed in $h$-PDMS, which avoids collapse of the structures while the bulk of the stamp consists of flexible PDMS to allow conformal contact between stamp and samples during the imprint process. The replicated grating samples are characterized by atomic force microscopy (AFM) and scanning electron microscopy (SEM); see Figs. 4(a) and $4(\mathrm{~b})$, where both the height and period of the patterns are found to be replicated with high fidelity. However, shrinkage of the gel during cross-linking introduces some edge roundness which may account for minor discrepancy between simulated and measured data.

Aside from the ease of structuring silica sol-gel via nanoimprint lithography, an important, additional advantage of the material is that pure silica nanostructures can be obtained by annealing it after crosslinking at $500^{\circ} \mathrm{C}$ in oxygen atmosphere [15]. This not only increases the mechanical strength of the silica layer but further promotes adhesion to the glass substrate. However, it should be noted that structural shrinkage takes place during annealing since all the organic constituents of the material are removed [28]. Consequently, the refractive index, period, and height of the grating lines are affected and must be considered during the design phase. In order to simplify the simulations of the fabricated grating structures, and to ease comparison of the optical measurements with the simulations, the annealing process has been omitted in this study such that the high fidelity between grating and master is maintained.

\section{Measurement Setup}

In order to characterize the optical daylighting performance of the gratings, the angular resolved transmitted diffraction efficiency is measured using a setup as shown schematically in Fig. 5(a). This setup allows measurement of continuous diffraction patterns over a $180^{\circ}$ span around the sample. Three lasers with wavelengths 410,532 , and $661 \mathrm{~nm}$

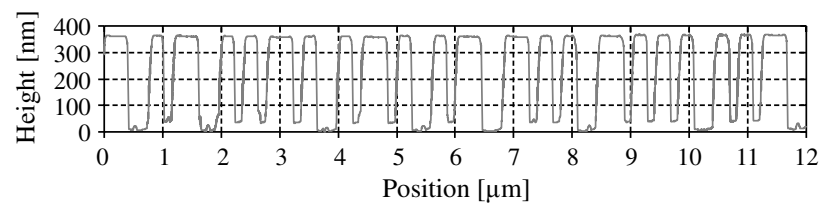

(a)

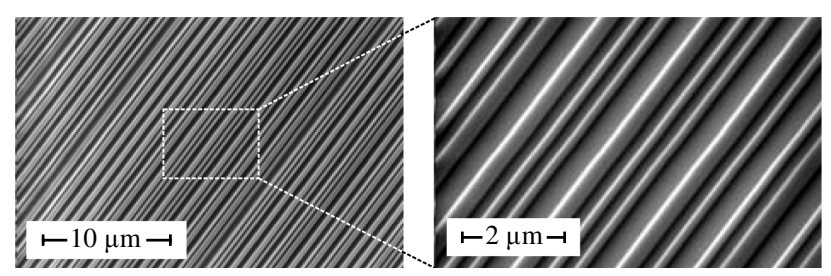

(b)

Fig. 4. (a) AFM-generated profile of a disordered grating $(M=2)$. (b) Top view SEM image of a disordered grating $(M=2)$. 


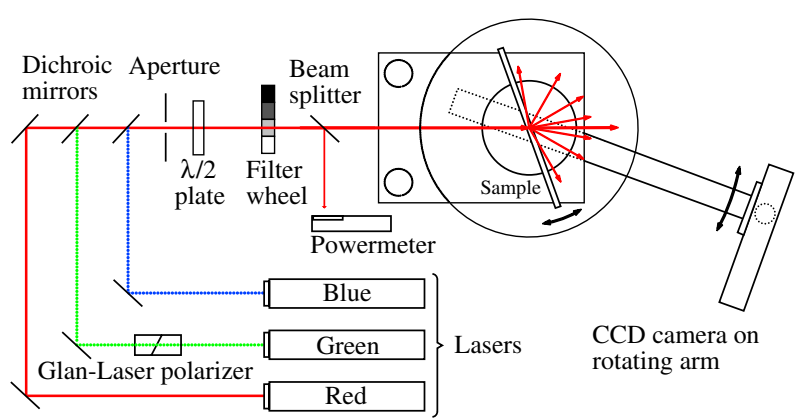

(a)

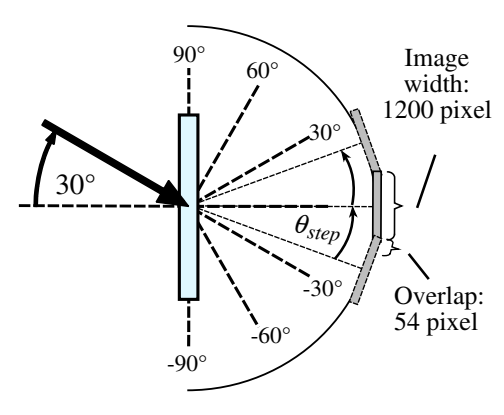

(b)

Fig. 5. (Color online) (a) Schematic of the measurement setup. Light from three lasers is combined into a single beam and directed at the sample under a fixed angle of incidence. A CCD camera rotates around the sample to measure the transmitted diffraction pattern. Each measurement is comprised of multiple images, taken with a small overlap. (b) Detail of the angle definitions. $0^{\circ}$ is defined as normal to the grating, positive angles correspond to light which is diffracted toward the ceiling, i.e., contributes to the daylighting.

(CrystaLaser DL405-25, CL532-025, and DL660-025, respectively) are used as light sources. For the green laser, a Glan-Laser polarizer is used to improve the polarization ratio such that all three lasers are $>100: 1$ linearly polarized. Even though the gratings are designed for application with incoherent, spectrally broad sunlight, the lasers offer the possibility to quantify the diffraction effect for distinct wavelength and polarization states. Similar to sunlight, the lasers have low divergence $(2 \mathrm{mrad})$. The three separate laser beams are combined using dichroic mirrors and then directed through an aperture to cut off non-Gaussian parts of the intensity profile. A broadband half-wavelength plate (Thorlabs AHWP05M-600) is used to control the polarization direction. A nonpolarizing beam splitter directs part of the beam to a power meter (Ophir NovaII with PD300 head), which serves as reference of the power incident on the sample. The sample is mounted on a rotation stage to control the angle of incidence, here fixed at $30^{\circ}$. See Fig. $5(\mathrm{~b})$ for a definition of the diffraction angles with respect to the grating. Measurement of the spatial distribution of the light transmitted through the sample is done with a CCD camera (Spiricon SP620U). The camera is mounted on an arm that rotates around the sample using a computer-controlled rotation stage (OWIS DMT100). As a result, the camera captures the complete diffraction pattern on a half circle around the grating. In order to operate the camera in a linear range, the measurement of the 0th order is attenuated by use of a ND3.0 neutral density absorption filter. The sensor active area height is $7 \mathrm{~mm}$ and the width is $5.3 \mathrm{~mm}$. In order to obtain a continuous image, the individual measurements are taken with a small overlap, mapped to a hemisphere, and then fitted together based on their cross-correlation [29]. In a typical measurement from $-90^{\circ}$ to $90^{\circ}$, the distance between camera and sample is $18 \mathrm{~cm}$, and the camera is rotated $1.5^{\circ}$ after each image, corresponding to an overlap of $\sim 54$ pixels between adjacent images. Thus the complete measurement consists of 120 images, each being $1200-54$ pixels wide, yielding an angular resolution of $764 \mathrm{pixel} /{ }^{\circ}$.

\section{Results and Discussion}

Figure 6 shows a measurement of the transmitted diffracted light for the sample with a $500 \mathrm{~nm}$ period and $25 \%$ disorder. The 0 th order, located at an angle of $-30^{\circ}$, has been attenuated by a factor of 1000 for better visibility of the diffracted light. The intensity of the red, green, and blue measurements are normalized and therefore not directly comparable, yet the figure still gives a reasonable qualitative representation of the daylighting effect for all three wavelengths. For $410 \mathrm{~nm}$ light, the angle of maximum efficiency is close to $15^{\circ}$, for $532 \mathrm{~nm}$ light the angle is close to $30^{\circ}$ and for $661 \mathrm{~nm}$ light close to $45^{\circ}$. The angular spread of the peaks is larger than $30^{\circ}$ and therefore yields a more homogeneous daylighting effect. Since coherent laser light is used for the measurement, the diffraction patterns also show fringes due to thin-film interference in the silica layer; see the enlarged region in Fig. 6 . These fringes would not be visible for incoherent sunlight, where instead a continuous light distribution would be present.

For a more detailed analysis of the diffraction efficiency, line traces are extracted from the images in the angular direction. Since the efficiency depends on the polarization of the light, both TE and TM polarization are measured and then averaged in order to mimic unpolarized sunlight. The result is

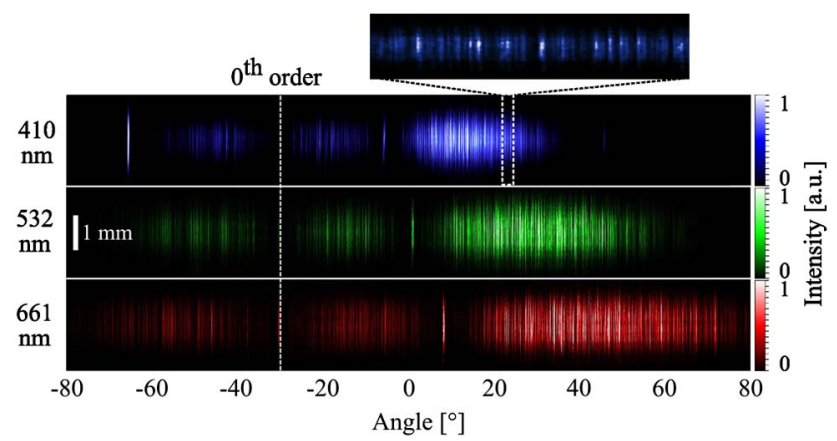

Fig. 6. (Color online) Transmitted diffraction pattern of a grating with $\Lambda=500$ and $M=2$, measured separately for 410,532 , and $661 \mathrm{~nm}$ TE polarized light at $30^{\circ}$ angle of incidence (artificial color). The 0th order, located at $-30^{\circ}$, has been attenuated by a factor of 1000 for better visibility of the diffracted light. 
shown in Fig. 7 for the four different grating geometries under $41 \overline{0}, 532$ and $661 \mathrm{~nm}$ light illumination, plotted together with the results obtained by the numerical RCWA simulations as described above. Again, it should be noted that the high frequency variation in the data is not caused by noise, but interference due to Fabry-Pérot resonances. Since this effect will not be present under sunlight illumination, it is not included in the simulation model. For the benefit of easier comparison between

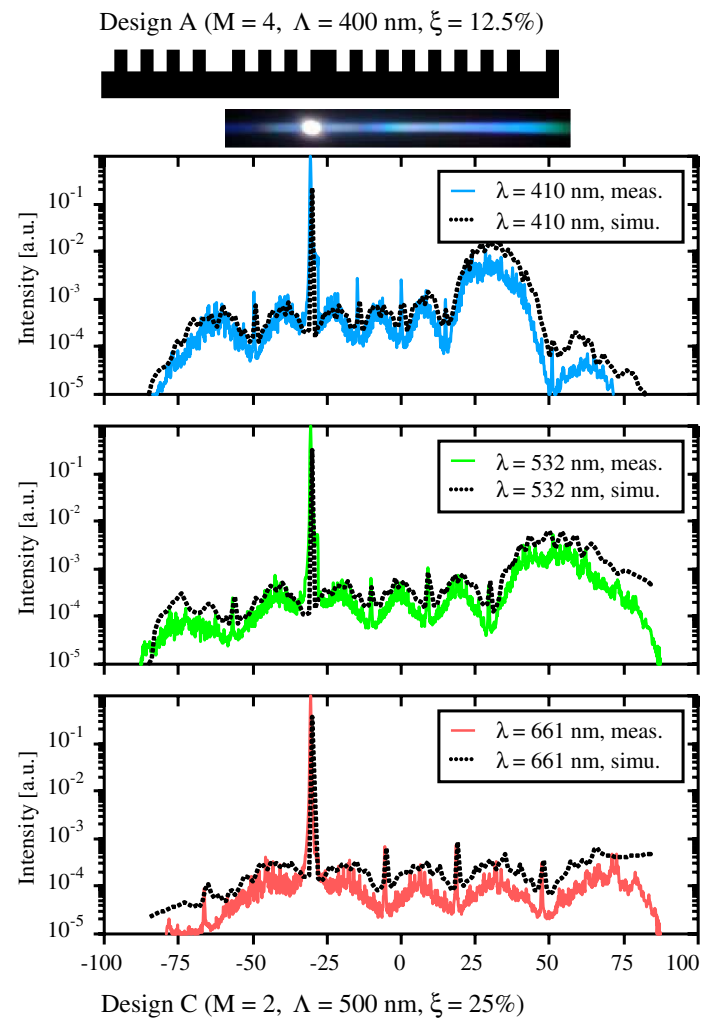

Design B (M =2, $\Lambda=400 \mathrm{~nm}, \xi=25 \%)$
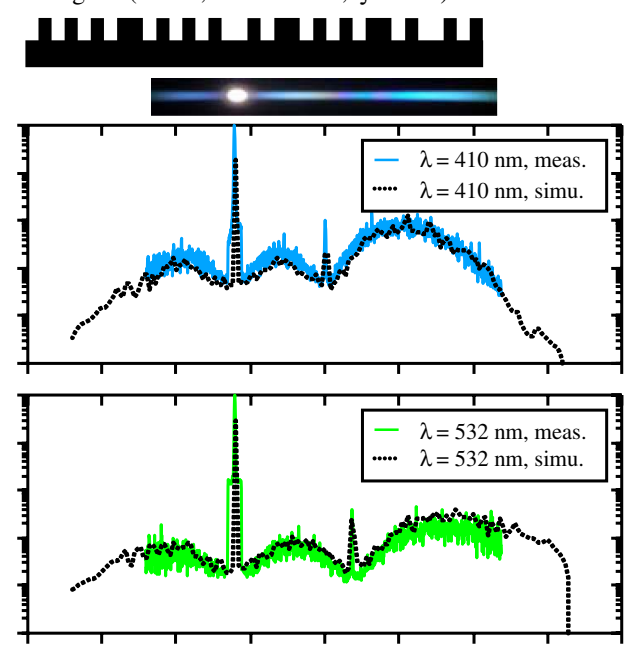

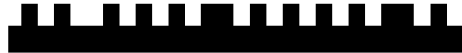
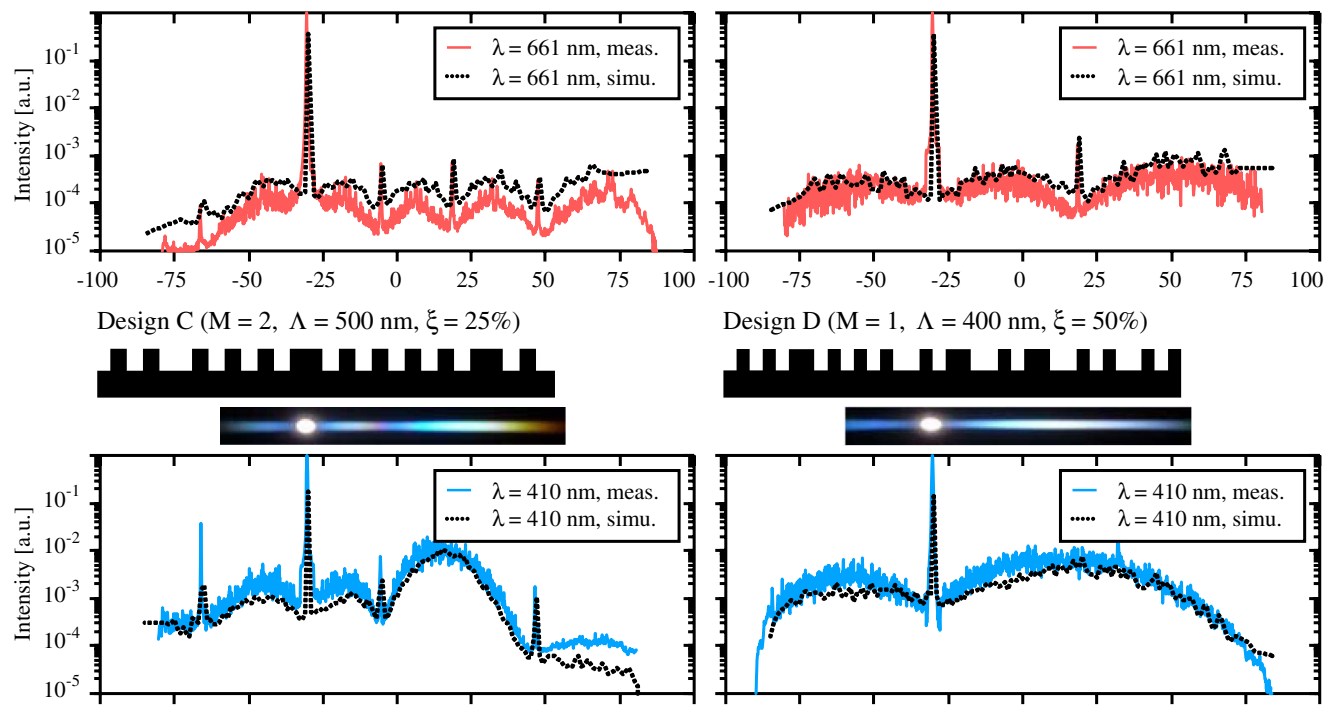

Design D ( $M=1, \Lambda=400 \mathrm{~nm}, \xi=50 \%)$
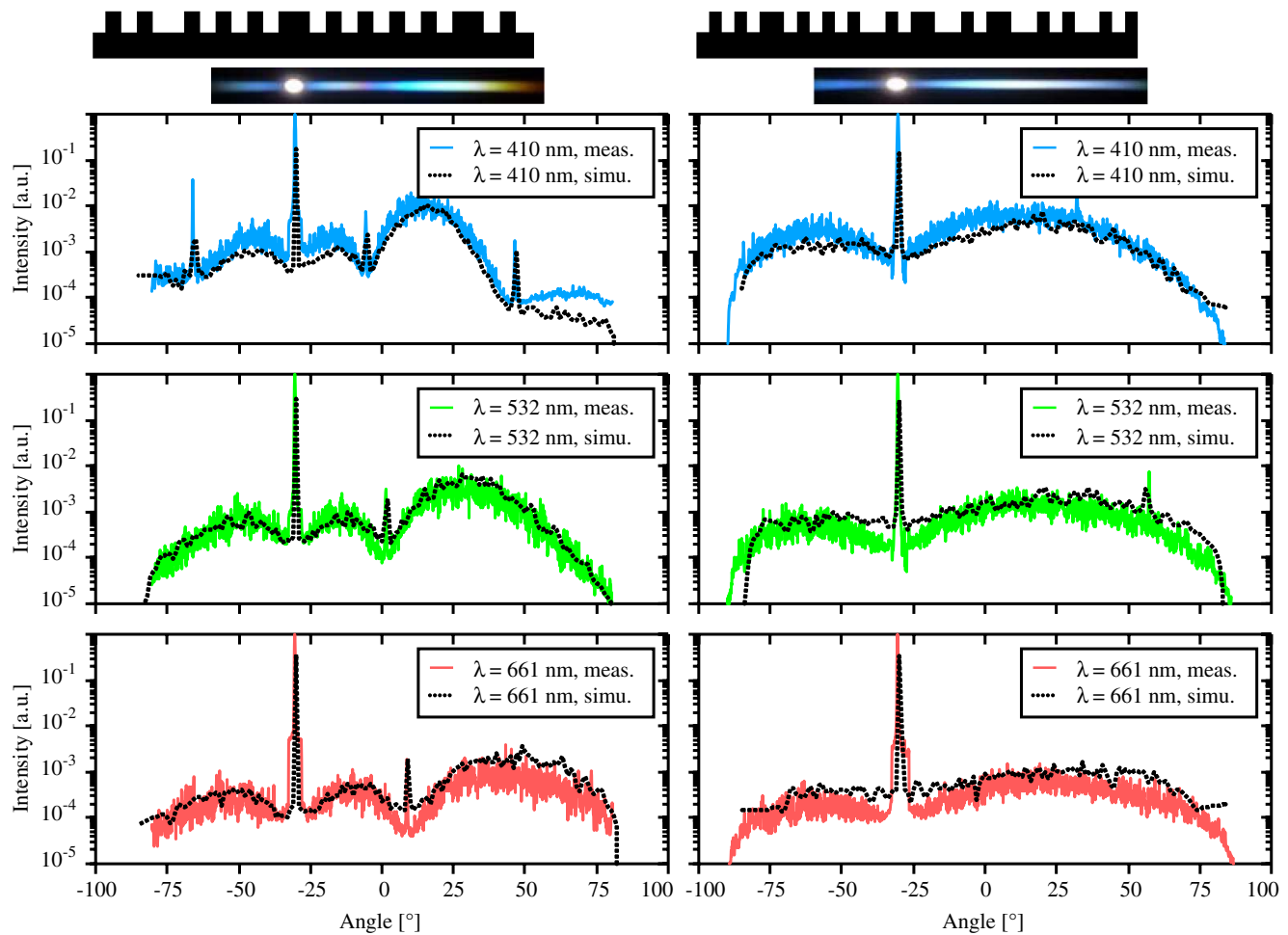

Fig. 7. (Color online) Curves showing the measured and simulated diffraction results for the four investigated samples at 410, 532, and $661 \mathrm{~nm}$. For each of the four samples, a drawing of the grating and a photograph of the diffraction pattern when illuminated with white xenon light is shown on top of the plots. 
Table 2. Measured and Simulated Daylighting Efficiencies of the Samples ${ }^{a}$

\begin{tabular}{|c|c|c|c|c|c|c|c|c|c|}
\hline \multirow[b]{2}{*}{ Period $[\mathrm{nm}]$} & \multirow[b]{2}{*}{$\xi[\%]$} & \multicolumn{4}{|c|}{ Efficiency [\%] (measurements) } & \multicolumn{4}{|c|}{ Efficiency [\%] (simulations) } \\
\hline & & $410 \mathrm{~nm}$ & $532 \mathrm{~nm}$ & $661 \mathrm{~nm}$ & Mean & $410 \mathrm{~nm}$ & $410 \mathrm{~nm}$ & $532 \mathrm{~nm}$ & Mean \\
\hline 400 & 12.5 & 29.5 & 11.6 & 3 & 11,4 & 48.6 & 29 & 5.7 & 28,0 \\
\hline 500 & 25 & 31.3 & 20.7 & 10.2 & 20,3 & 43.4 & 32.3 & 18.7 & 31,7 \\
\hline 400 & 50 & 28.7 & 15.7 & 8.2 & 15,5 & 42.3 & 26.5 & 14.6 & 26,1 \\
\hline
\end{tabular}

${ }^{a}$ The mean value is weighted with the spectral sensitivity of the human eye, assumed as $0.012,0.925$, and 0.053 for 410,532 , and $661 \mathrm{~nm}$, respectively [31].

measurements and simulated data, a $0.1^{\circ}$ wide moving average filter is used to suppress these resonances.

The general trend of the curves shows clear qualitative agreement between simulation and experiment, in particular validating the SSGNIL process as adequately capable of replicating the nanoscale period and high aspect ratio grating structures. As expected, the sample with the lowest degree of disorder (design A, $M=4, \xi=12.5 \%$ ) shows the most prominent peaks in the diffraction spectrum. Apart from several broad peaks, very sharp peaks are also observed (e.g., at $-5^{\circ}, 19^{\circ}$, and $48^{\circ}$ ). The positions of these sharp peaks coincide with the diffraction orders of a hypothetical grating of uniform $M \cdot \Lambda=$ $800 \mathrm{~nm}$ periodicity, indicating that the peaks are introduced by the randomization method. By increasing the disorder (design $\mathrm{B}, M=2, \xi=25 \%$ ), the number of peaks is reduced, such that the light is redirected into significantly smoothened, broader maxima, and a single sharp peak. The comparison between the base periods of 400 and $500 \mathrm{~nm}$ shows that the longer period causes the maxima to separate less and move to smaller angles, similar to a uniform grating. When the disorder is increased further to $50 \%(M=1)$, the redirected light distributes evenly over the range of $-25^{\circ}$ to $75^{\circ}$ for all the three wavelengths. Since the randomization occurs after each period, no sharp peaks exist in this case. Figure 7 also shows for each of the four gratings a photograph of the diffraction pattern when the grating is illuminated with white light from a xenon lamp. Here it is seen that design A shows a rainbow effect since the redirection of the different wavelengths does not overlap. In design $B$ the maxima are broad and homogeneous, but the rainbow effect is more pronounced due to the diffraction angle of the sharp peak being wavelength dependent (located at $0.1^{\circ}$, $9.2^{\circ}$, and $18.5^{\circ}$ ). The most prominent color divergence is seen in design $\mathrm{C}$ with its larger period of $500 \mathrm{~nm}$, especially at high angles where blue light is hardly diffracted to, but a significant amount of red light is present. Finally, sample D shows broad redirection without sharp peaks and therefore yields a highly homogeneous white light diffraction pattern, making this sample most suitable for daylighting.

The daylighting efficiency $\eta_{\text {daylighting }}$ is calculated from the measurements and summarized in Table 2. Typically for all samples, the highest efficiencies of approximately $30 \%$ are achieved with blue light. The efficiency is lower for red and green light (3\%-15\%) which is, however, not a problem, since there is often a lack of short wavelength light in indoor lighting [30].

By adjusting the period from 400 to $500 \mathrm{~nm}$, it is possible to increase the efficiency for green light up to $21 \%$ and red light up to $10 \%$. Doing so, of course, reduces the amount of light in the 0th order, leading to a decisional compromise between transparency and daylighting effect.

Even though the simulated shape of the curves is closely reproduced in the experiments, the efficiency of the measurements is $40 \%-60 \%$ lower than predicted by the simulations. This deviation is attributed to two factors. First, fabrication deficiencies, where incomplete replication of thin grating lines are observed, and AFM measurements reveal line roughness and variation in grating heights. The fabrication factors can be overcome by fine-tuning the imprinting parameters. Second, mechanical damage of the gratings after fabrication decreases the overall efficiency. In order to overcome this problem, the mechanical stability of the silica film can be increased by using a hard-bake as mentioned in Section 4 . For daylighting applications, the nanostructures could be protected from mechanical damage by positioning them on the inside of a double glazing.

The measurements indicate that the efficiency is mostly independent on the disorder, pointing to the use of varying the degree of disorder to tune the angular distribution of the light without affecting the efficiency. Accordingly, this enables the scheme to fulfill the requirements of specific applications, determined, e.g., by room size, purpose, or trajectory of the sun. As a result, two major problems that exist for daylighting applications have been solved: (1) fabrication of optical nanostructures in a UV-stable material rather than polymer films and (2) suppression of the "rainbow effect" via the use of disordered gratings. Since the optical effect is achieved with a thin-film grating, the approach can be applied to a wide variety of planar and nonplanar substrates for applications where tailored diffraction patterns are required.

\section{Conclusion}

In conclusion, nanoscale disordered gratings are demonstrated to enable controlled diffraction of light into broad distributions over large angular range, thus adding new optical functionality to transparent substrates. It is shown that the fabrication of these gratings is feasible with both high structural fidelity 
and adhesion to glass by using sol-gel nanoimprint lithography. Considering established methods to remove the organic compounds from the sol-gel silica via thermal annealing such that pure silica resides, the proposed scheme not only points to cost-effective fabrication over large surfaces but also long-term UV-light stability. For specific designs of disordered gratings targeted toward daylighting applications on window glasses, optimal parameters are determined by numerical RCWA simulations, namely $350 \mathrm{~nm}$ height and $400 \mathrm{~nm}$ period. In order to validate the design and fabrication process, an optical setup is developed, capable of measuring the complete continuous diffraction patterns at high angular resolution of 760 samples $/{ }^{\circ}$. Gratings with various degrees of disorder are investigated, and comparing the measurement results with simulations confirms that the diffraction characteristics of the disordered gratings can be predicted. Average measured daylighting efficiencies for $500 \mathrm{~nm}$ gratings are $20 \%$ and for

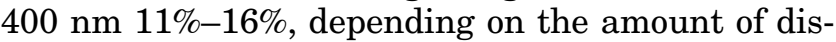
order. Since the degree of disorder has only small influence on the efficiency, the disorder can be modified to tailor the diffraction between directional and widely distributed patterns. This feature renders the disordered gratings well suited to daylighting and other applications that require customizable light redirection over broad angular range.

This work was funded as part of the EC project NaPaNIL (Contract No. 214249). The authors acknowledge help from U. Plachetka and F. Schlachter for $h$-PDMS fabrication (AMO), S. Landis for master fabrication (CEA Leti), and N. Chemin for NIL (SGR). The authors wish to thank P. Lalanne (LCFIO) for helping us define the grating structures. C.L.C. Smith is supported by an EU FP7 Marie Curie Fellowship (project number PIIF-GA-2009-254573) and the Danish Research Council for Technology and Production Sciences (grant number 12-126601).

\section{References}

1. D. Meyerhofer, "Spatial resolution of relief holograms in dichromated gelatin," Appl. Opt. 10, 416-421 (1971).

2. S. T. Han, Y.-L. Tsao, R. M. Walser, and M. F. Becker, "Electromagnetic scattering of two-dimensional surfacerelief dielectric gratings," Appl. Opt. 31, 2343-2352 (1992).

3. X. Sheng, S. G. Johnson, J. Michel, and L. C. Kimerling, "Optimization-based design of surface textures for thin-film Si solar cells," Opt. Express 19, A841-A850 (2011).

4. S. Singh, "Diffraction gratings: aberrations and applications," Opt. Laser Technol. 31, 195-218 (1999).

5. S. Ura, Y. Furukawa, T. Suhara, and H. Nishihara, "Linearly focusing grating coupler for integrated-optic parallel pickup," J. Opt. Soc. Am. A 7, 1759-1763 (1990).

6. R. Shechter, Y. Amitai, and A. A. Friesem, "Compact beam expander with linear gratings," Appl. Opt. 41, 1236-1240 (2002).

7. P. Mouroulis, D. W. Wilson, P. D. Maker, and R. E. Muller, "Convex grating types for concentric imaging spectrometers," Appl. Opt. 37, 7200-7208 (1998).

8. P. Licinio, M. Lerotic, and M. S. S. Dantas, "Diffraction by disordered gratings and the Debye-Waller effect," Am. J. Phys. 67, 1013-1016 (1999).

9. J. M. Rico-García and L. M. Sanchez-Brea, "Binary gratings with random heights," Appl. Opt. 48, 3062-3069 (2009).
10. A. Oskooi, P. A. Favuzzi, Y. Tanaka, H. Shigeta, Y. Kawakami, and S. Noda, "Partially disordered photonic-crystal thin films for enhanced and robust photovoltaics," Appl. Phys. Lett. 100, 181110-181114 (2012).

11. B. Bläsi, A. Gombert, and M. Niggemann, "Microstructured polymer surfaces with complex optical functions for solar applications," in Polymers-Opportunities and Risks II SE-14, P. Eyerer, M. Weller, and C. Hübner, eds. (Springer, 2009), Vol. 12, pp. 263-279.

12. S. Klammt, A. Neyer, and H. F. O. Müller, "Microoptics for efficient redirection of sunlight," Appl. Opt. 51, 2051-2056 (2012).

13. O. Masuda and S. M. C. Nascimento, "Lighting spectrum to maximize colorfulness," Opt. Lett. 37, 407-409 (2012).

14. R. H. Katyl, "Moiré screens coded with pseudo-random sequences," Appl. Opt. 11, 2278-2285 (1972).

15. C. Peroz, C. Heitz, E. Barthel, E. Søndergård, and V. Goletto, "Glass nanostructures fabricated by soft thermal nanoimprint,” J. Vac. Sci. Technol. B 25, L27-L30 (2007).

16. C. Gourgon, A. K. Ferchichi, D. Pietroy, T. Haatainen, and J. Tesseire, "Scatterometry analysis of sequentially imprinted patterns: influence of thermal parameters," Microelectron. Eng. 98, 270-274 (2012).

17. H. D. Tholl, C. G. Stojanoff, R. Kubiza, and G. Willbold-Lohr, "Design optimization and manufacturing of holographic windows for daylighting applications in buildings," Proc. SPIE 2017, 35-45 (1993).

18. C. L. Robbins, Daylighting: Design and Analysis (Van Nostrand Reinhold, 1986).

19. M. Kischkoweit-Lopin, "An overview of daylighting systems," Sol. Energy 73, 77-82 (2002).

20. R. G. Hopkinson, "Glare from daylighting in buildings," Appl. Ergon. 3, 206-215 (1972).

21. S. W. Lockley, G. C. Brainard, and C. A. Czeisler, "High sensitivity of the human circadian melatonin rhythm to resetting by short wavelength light," J. Clin. Endocrinol. Metab. 88, 4502-4505 (2003).

22. M. F. Lewis and C. L. West, "Some focusing properties of chirped gratings," Opt. Quantum Electron. 21, 17-33 (1989).

23. M. G. Moharam, E. B. Grann, D. A. Pommet, and T. K. Gaylord, "Formulation for stable and efficient implementation of the rigorous coupled-wave analysis of binary gratings," J. Opt. Soc. Am. A 12, 1068-1076 (1995).

24. P. Lalanne and G. M. Morris, "Highly improved convergence of the coupled-wave method for TM polarization," J. Opt. Soc. Am. A 13, 779-784 (1996).

25. C. Peroz, V. Chauveau, E. Barthel, and E. Søndergård "Nanoimprint lithography on silica sol-gels: a simple route to sequential patterning," Adv. Mater. 21, 555-558 (2009).

26. A. Letailleur, J. Teisseire, N. Chemin, E. Barthel, and E. Søndergård, "Chemorheology of sol-gel silica for the patterning of high aspect ratio structures by nanoimprint," Chem. Mater. 22, 3143-3151 (2010).

27. H. Schmid and B. Michel, "Siloxane polymers for highresolution, high-accuracy soft lithography," Macromolecules 33, 3042-3049 (2000).

28. M. B. Mikkelsen, A. A. Letailleur, E. Søndergård, E. Barthel, J. Teisseire, R. Marie, and A. Kristensen, "All-silica nanofluidic devices for DNA-analysis fabricated by imprint of sol-gel silica with silicon stamp," Lab Chip 12, 262-267 (2011).

29. R. Maurer, F. Schneider, C. Vogt, M. Schinhaerl, P. Sperber, and R. Rascher, "Physical marker based stitching process of circular and non-circular interferograms," Proc. SPIE 8083, 80830Q (2011).

30. M. G. Figueiro and M. S. Rea, "Lack of short-wavelength light during the school day delays dim light melatonin onset (DLMO) in middle school students," Neuroendocrinol. Lett. 31, 92-96 (2010)

31. L. T. Sharpe, A. Stockman, W. Jagla, and H. Jägle, "A luminous efficiency function, VD65* $(\lambda)$, for daylight adaptation: a correction," Color Res. Appl. 36, 42-46 (2011).

32. K. Papamichael, C. Ehrlich, and G. Ward, "Design and evaluation of daylighting applications of holographic glazings," Final Report prepared for Physical Optics Corporation under Contract Agreement Number BG-95037 (1996). 